\title{
Diffractive Phenomena at Tevatron ${ }^{1}$
}

\author{
A. Santoro ${ }^{2}$ \\ For the DØ Collaboration \\ $\mathrm{LAFEX} / \mathrm{CBPF}$ \\ Rio de Janeiro, RJ, Brazil \\ Fermilab \\ P.O.Box 500, Batavia, Il \\ 60510, USA
}

\begin{abstract}
.
Preliminary results from the $\mathrm{D} \varnothing$ experiment on jet production with rapidity gaps in $p \bar{p}$ collisions are presented. A class of dijet events with a forward rapidity gap is observed at center-of-mass energies $\sqrt{s}=1800 \mathrm{GeV}$ and 630 $\mathrm{GeV}$. The number of events with rapidity gaps at both center-of-mass energies is significantly greater than the expectation from multiplicity fluctuations and is consistent with a hard single diffractive process. A class of events with two forward gaps and central dijets are also observed at $1800 \mathrm{GeV}$. This topology is consistent with hard double pomeron exchange. We also present proposed plans for extending these analysis into Run II through the use of a forward proton detector.
\end{abstract}

\section{INTRODUCTION}

The results coming from Tevatron (CDF and DØ) and Hera (H1 and ZEUS) $[1,2]$ and the progress in the phenomenological and theoretical side of diffractive physics are very important to understand the complexity of the pomeron in high energy physics. Soft and hard diffraction are an important part of strong interactions. Soft diffraction is well described phenomenologically by the Regge Model (RM) with a hadronic pomeron [3]. For hard diffraction [4] we have many interesting models proposing different configurations for the pomeron as a composite object. Among the leading models for a hard pomeron we have the "economical" two-gluon model [5] and the "hot spot of gluons" or the BFKL pomeron [6].

Hard diffraction can be best studied at the Tevatron due to the large diffractive mass accessible. For hard single diffraction we have aproximately $\mathrm{M}_{\mathrm{x}}=400$ $\mathrm{GeV}$ and for double pomeron exchange $\mathrm{M}_{\mathrm{x}}=100 \mathrm{GeV}$, where $\mathrm{M}_{\mathrm{x}}$ is the diffractive mass produced by interactions with pomeron. We believe that these subjects are certainly a good bridge between the soft (RM) and hard (QCD) [4] interactions. We will present here some preliminary results from $\mathrm{D} \varnothing$ for hard single diffraction and for double pomeron exchange [2].

1) Published Proceedings from VI Conference on the Intersections of Particle and Nuclear physics, Big Sky, Montana, USA, May 27-June 21997.

2) santoro@lafex.cbpf.br 


\section{HARD SINGLE DIFFRACTION}

An experimental signature of hard diffractive events is the presence of a rapidity gap (lack of particle production in a rapidity or pseudorapidity ${ }^{3}$ region), along with a hard scattering (jet production, $W$ production, etc.). Since the pomeron is a color singlet, radiation is suppressed in events with pomeron exchange, typically resulting in a large rapidity gap [9]. In hard single diffraction a pomeron is emitted from one of the incident particles (proton or antiproton) and undergoes a hard scattering with the second proton, often leaving a rapidity gap in the direction of its parent particle (antiproton or proton). We examine the process $p+\bar{p} \rightarrow j+j+X$ and look for the presence of a forward rapidity gap along the direction of one of the initial beam particles.

The existence of a diffractive signal in the experimental data may be observed as a larger number of rapidity gap events in the forward multiplicity distribution than expected from the non-diffractive background. Given sufficient detector resolution, sensitivity, and statistics, two components in the multiplicity distribution can be resolved and the relative fraction of rapidity gap events in excess of expectations from a smoothly falling multiplicity distribution can be estimated.

The $\mathrm{D} \varnothing$ detector [7] is used to provide experimental information on the fraction of jet events with forward rapidity gaps. This analysis primarily utilizes the uranium-liquid argon calorimeters which have full coverage for a pseudorapidity range of $|\eta|<4$.1. The transverse segmentation of the projective calorimeter towers is typically $\Delta \eta \times \Delta \phi=0.1 \times 0.1$. The electromagnetic (EM) section of the calorimeters is used to search for rapidity gaps. The EM section is particularly useful for identifying low energy particles due to its low level of noise and ability to detect neutral pions. A particle is tagged by the deposition of more than $200 \mathrm{MeV}$ of energy in a single EM calorimeter tower.

The data used in this study were obtained using a forward trigger requiring at least two jets above $12 \mathrm{GeV}$ in the same hemisphere with both jets having $\eta>1.6$ or $\eta<-1.6$. Since the pomeron only carries a few per cent of the initial proton momentum, the jet system is expected to be boosted in diffractive jet production, thus a forward jet trigger can be utilized to provide an enhanced sample of diffractive events. Offline, two jets above trigger threshold are required and events with multiple $p \bar{p}$ interactions or spurious jets are removed. Jets are reconstructed using a cone algorithm with radius, $R=\sqrt{\Delta \eta^{2}+\Delta \phi^{2}}=0.7$. The number of EM towers $\left(\mathrm{n}_{\mathrm{EM}}\right)$ above a $200 \mathrm{MeV}$ energy threshold is measured in the hemisphere opposite the leading two jets in the region $2<|\eta|<4$.1. The $\left(\mathrm{n}_{\mathrm{EM}}\right)$ distribution for the forward trigger is shown in Fig. 1 for $\sqrt{s}$ of (a) $1800 \mathrm{GeV}$ and (b) $630 \mathrm{GeV}$.

3) Pseudorapidity or $\eta=-\ln \left[\tan \left(\frac{\theta}{2}\right)\right]$, where $\theta$ is the polar angle defined relative to the proton beam direction. 

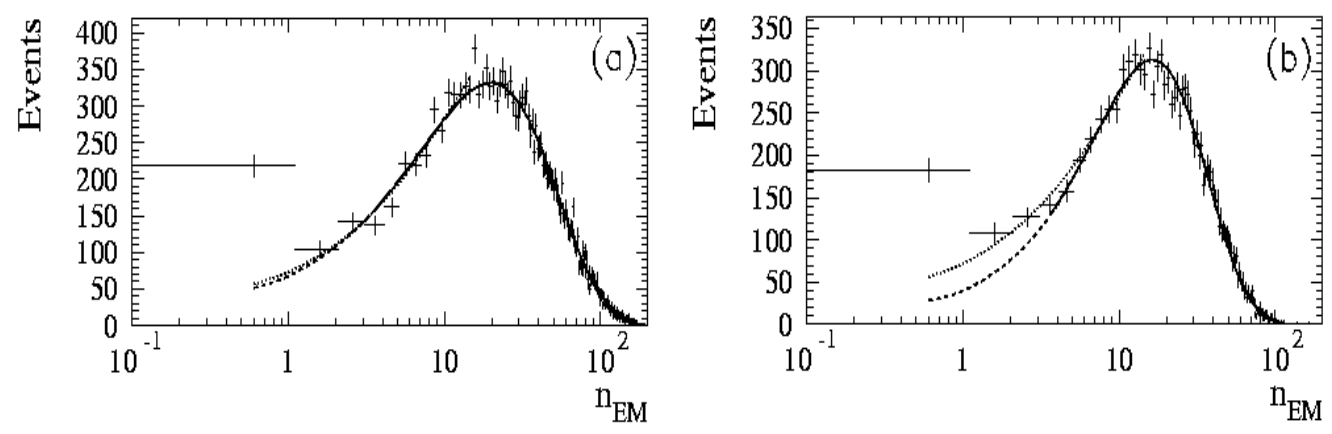

FIGURE 1. Number of electromagnetic calorimeter towers $\left(\mathrm{n}_{\mathrm{EM}}\right)$ above a $200 \mathrm{MeV}$ energy threshold for the region $2<\eta<4.1$ opposite the forward jets for center-of-mass energies of (a) $1800 \mathrm{GeV}$ and (b) $630 \mathrm{GeV}$. The curves are negative binomial fits to the data excluding low multiplicity bins.

The distributions at both center-of-mass energies show a peak at zero multiplicity in qualitative agreement with expectations for a diffractive signal component. Negative binomial fits to the leading edge and the whole distribution (excluding $\mathrm{n}_{\mathrm{EM}}=0$ ) have been used to estimate the non-diffractive background. A fractional excess of rapidity gap events is defined to be the number of zero multiplicity events in excess of those predicted by the fit divided by the total number of events in the sample. The fractional excess observed in the forward region for the $\sqrt{s}=1800 \mathrm{GeV}$ sample is $0.67 \pm 0.05 \%$, where the error includes only statistical uncertainties and a systematic uncertainty based on the choice of range for the fit. An excess of rapidity gap events is also clearly observed at $630 \mathrm{GeV}$ with a magnitude of $1-2 \%$. Systematic studies have not been completed, but effects such as gap detection efficiency are expected to reduce the number of observed rapidity gaps, and correcting for these effects is expected to give a modest increase in the magnitude of the signal measurement. The observed fractional excess is relatively insensitive to the calorimeter energy threshold, and rapidity gap events $\left(n_{\mathrm{EM}}=0\right)$ typically have zero multiplicity in other available detectors, such as hadronic calorimeters, forward tracking, beam hodoscopes, and forward muon chambers.

The forward gap fraction measurement for the $\sqrt{s}=1800 \mathrm{GeV}$ sample has been extended to unrestricted jet topologies using an inclusive jet trigger and we observe that the gap fraction increases with the boost of the jets, consistent with the expected behavior of diffractive events discussed earlier.

\section{DOUBLE POMERON EXCHANGE}

The same experimental methods may be applied to a search for hard double pomeron exchange. In this process both incoming protons emit a pomeron and the two pomerons interact to produce a jet system. Rapidity gaps are 
expected to be produced along each forward beam direction, since there is no color connection between the jet system and the beam particles. In this analysis we have selected an enhanced sample of forward rapidity gap events with a dedicated single gap trigger, which, in addition to the jet requirements, vetoes on forward particles in either beam direction using the scintillator beam hodoscopes which bracket the $\mathrm{D} \varnothing$ collision region. Events were selected to have a rapidity gap $\left(\mathrm{n}_{\mathrm{EM}}=0\right)$ in the direction of the online veto. These data consist of about 40,000 single gap events at $\sqrt{s}=1800 \mathrm{GeV}$, compared to the approximately 200 events observed in the forward trigger sample after background subtraction. This enhanced diffractive sample is used to search for double forward gap events, in which we require no towers above threshold in both forward calorimeter regions along with two jets with $E_{T}>15 \mathrm{GeV}$ and $|\eta|<1.0$. This is an expected topology for events produced in hard double pomeron exchange. The $n_{\mathrm{EM}}$ distribution for the veto-trigger is plotted in Fig. 2 for the forward region $(2<|\eta|<4.1)$ opposite the tagged rapidity gap. We clearly observe a sample of double gap events, although an interpretation of them in terms of hard double pomeron exchange requires further study.

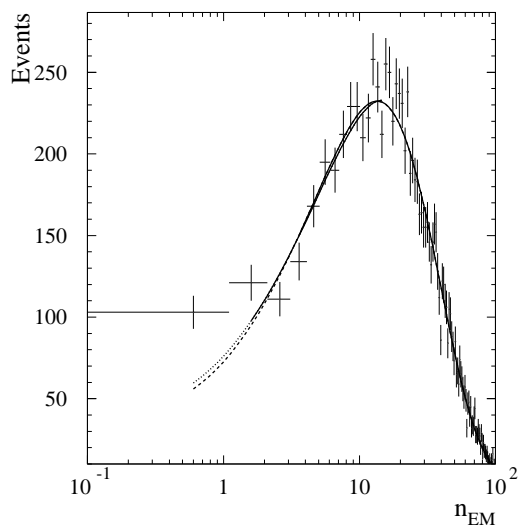

FIGURE 2. The $\mathrm{n}_{\mathrm{EM}}$ distribution opposite the tagged gap for single gap trigger data. The zero multiplicity events are double gap events in this sample. The curves are negative binomial fits to the data excluding low multiplicity bins as described in the previous section.

\section{CONCLUSION AND PROSPECTS FOR THE NEXT FUTURE -RUN II AT THE TEVATRON}

We have observed the presence of forward rapidity gaps in events with high $E_{T}$ jet production with the D $\varnothing$ detector at Fermilab. The fraction of forward rapidity gap events observed is in excess of those expected to be produced via multiplicity fluctuations at center-of-mass energies of $1800 \mathrm{GeV}$ and $630 \mathrm{GeV}$. This is consistent with expectations from hard single diffractive jet production and provides the first experimental evidence for this process at $\sqrt{s}=1800 \mathrm{GeV}$. We also observe a class of events containing high $E_{T}$ central 
jets and two forward rapidity gaps, consistent with a hard double pomeron exchange event topology.

All these results motivated us to propose a new set of sub-detectors (the so called Roman Pots [8]) to be introduced in the two arms of the D $\varnothing$ Detector. This forward proton detector (FPD) is composed of four quadrupole spectrometers and one dipole spectrometer. They will tag the scattered proton and/or anti-proton, allowing us to observe directly a number of processes like double pomeron exchange and improve our studies of all diffractive topics in Run II at the Tevatron.

We acknowledge the support of the US Department of Energy and the collaborating institutions and their funding agencies in this work.

\section{REFERENCES}

1. A. Brandt et al. (UA8 Collaboration), Phys. Lett. B 297, 417 (1992); S. Abachi et al.(DØ Collaboration) Phys. Rev. Lett.76,734 (1996); P. Melese (CDF Collaboration), Proceedings of the 11th Topical Workshop on Proton-Antiproton Collider Physics, Abano Terme, Italy, 1996; A. Doyle, Workshop on HERA Physics, "Proton, Photon, and Pomeron Structure", GLAS-PPE/96-01; F. Abe et al. (CDF Collaboration), Phys. Rev. Lett. 78 (1997) 2698.

2. A. Brandt (DØ Collaboration) Proceedings of the 11th Topical Workshop on Proton-Antiproton Collider Physics, Abano Terme, Italy, 1996.

3. E. L. Berger and P. Pirila, Phys.Rev. D12,3448 (1975); Phys.Lett. 59B,361 (1975); E. L. Berger and R. Cutler, Phys.Rev. D15, 1903 (1977); G. CohenTannoudji, A. Santoro and M. Souza, Nucl.Phys. B125, 445 (1977); G. Alberi and G. Goggi, Phys.Report 74,1 (1981); K. Goulianos Phys.Report 3,169 (1983); A. Donnachie and P. V. Landshoff, Phys.Lett. B296,227 (1992); S. V. Levonian 28th.ICHEP Proceedings vol.I,17 Warsaw (1996)

4. G. I. Ingelman and P. E. Schlein, Phys. Lett. 152B,256 (1985); E. L. Berger, J. C. Collins, D. E. Soper, G. Sterman, Nucl.Phys. B286,704 (1987); G. Sterman, 28th. ICHEP Proceedings, vol.I,103 (1996)

5. S. Nussivov, P.R.L. 34,1286 (1975);P.R. D14,246 (1976); F. E. Low, P.R. D12,163 (1975); J. D. Bjorken, P.R. D47,101 (1993)

6. Ia. Ia. Balitski, L. N. Lipatov, Sov. J. Nucl. Phys. 28,822 (1978); E. A. Kuraev, L. N. Lipatov, V. S. Fadin, Sov. Phys. JETP 45,199 (1977);

7. S. Abachi et al. (DØ Collaboration), Nucl. Instrum. and Meth. in Phys. Res. A 338, 185 (1994).

8. G. Matthiae, Rep. Prog. Phys. 57,743 (1994).

9. H. Chehime and D. Zeppenfeld, preprint $M A D / P H / 814$ (1994). 